\title{
The Stress's State Analysis of Carbon Fibre Reinforced Concrete Elements Evaluating the Bond Influence
}

\section{Tadas Lisauskas, Mindaugas Augonis, Šarūnas Kelpša}

Kaunas University of Technology, Faculty of Civil Engineering and Architecture Studentu st. 48, LT-51367 Kaunas, Lithuania

*Corresponding author: tadaslisauskas@gmail.com

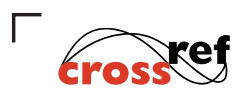

http://dx.doi.org/10.5755/j01.sace.23.2.20987
While strengthening structures with thin layered elements an important role is carried out by the bond between separate elements, but there are not any methods which would describe the distribution of bond stress in the contact zone when the concrete behaviour is elastic and plastic. In this article a single span reinforced concrete beam which is strengthened by CFRP (Carbon fibre reinforced polymer) is analysed and three methods which could describe bond stress through the length of the element are provided. The three compared methods are: finite elements method (FEM), theory of multiple rods and the proposed method of the authors. The similarities and differences of results are shown by diagrams and discussed. The method that the authors propose is superior to other methods because of evaluation of the plastic behaviour of concrete which is not possible to evaluate it by the method of the theory of multiple rods.

Keywords: bond, CFRP, reinforced concrete, stress, strengthening.

Nowadays in modern world, when architecture is getting more complex and the structural requirements are growing higher, the strengthening of structures plays an important role. In this article the strengthening of reinforced concrete beam structures with thin layered materials is analysed. The main focus is given to analyse the contact zone between separate layers. Many authors analyse the contact zone between fibre and concrete, but these methods separately not evaluate the effect of concrete behaviour in reinforced concrete structures. In this article the analysis of reinforced flexural reinforced concrete structures is made, applying the finite elements method, the theory of multiple rods method and the method proposed by the authors.

The experiments show that the highest bond stress in the contact zone was at the front and the back end of the beam. However, when the load is increasing, the bond at the end of a beam is decreasing, so the highest bond stress shifts a little bit further from the end. It happens because at the end of the beam the tension strength of a concrete is exceeded. (Guo2005, Guo 2007)

While analysing a single span beam which is strengthened by CFRP it was received that the shear contact stress increases evenly at the centre of a beam and increases greatly at the ends. (Lorenzis 2001)

\section{Introduction}

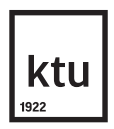

Journal of Sustainable Architecture and Civil Engineering Vol. 2 / No. 23 / 2018 pp. $79-10$ DOI 10.5755/j01.sace.23.2.20987 
Methods

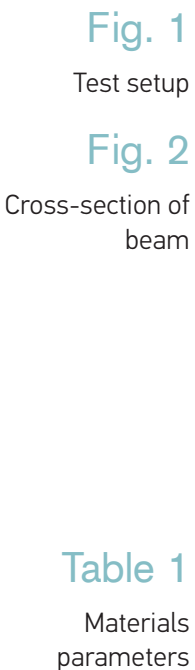

\section{Fig. 3}

Distribution of shear stress at the ends of the beam
The examined composite beams are made of two elements so the interaction between the elements is analysed when there are different bonds in the elastic stage because of a hydrothermal load. To describe the bond between different elements the theory of multiple rods is being used. (Zabulionis 2005).

In this article a single span reinforced concrete beam reinforced by carbon fibre is analysed. The test setup and the cross-section of the beam are provided in the figures Fig. 1 and Fig. 2. Concrete, steel, carbon fibre and glue material parameters are provided in the Table 1.
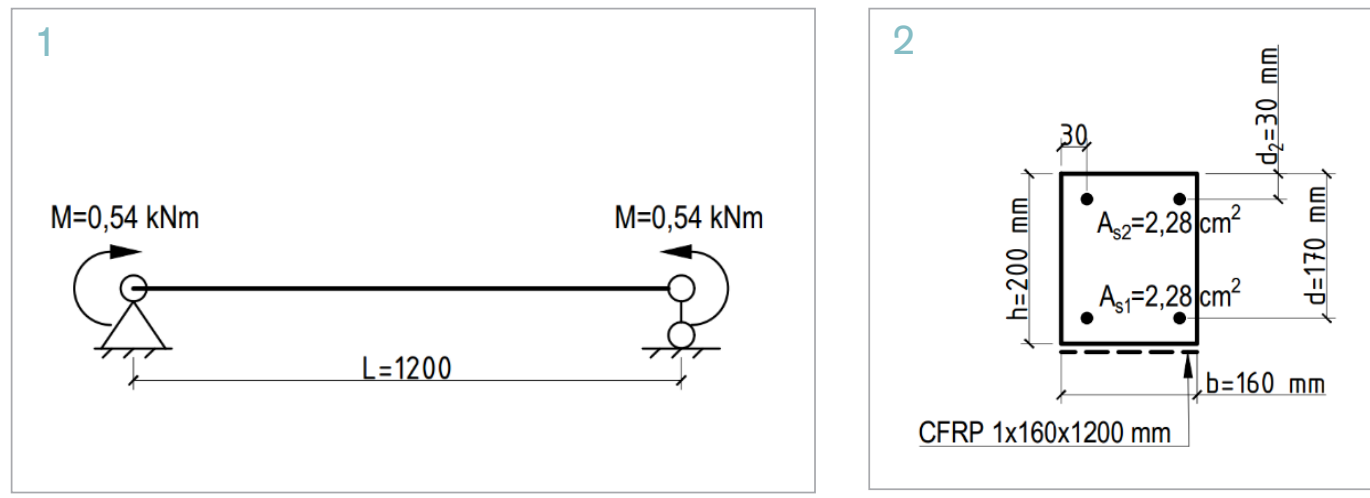

\begin{tabular}{c|c|c|c|c|c|c}
\hline$E_{c}$ & $E_{s}$ & $E_{\text {CFRP }}$ & $U_{c}$ & $U_{s}$ & $U_{g}$ & $U_{\text {CFRP }}$ \\
\hline $28 \mathrm{GPa}$ & $200 \mathrm{GPa}$ & $230 \mathrm{GPa}$ & 0,25 & 0,3 & 0,3 & 0,3 \\
\hline
\end{tabular}

Where: $E_{c}, E_{s}, E_{C F R P}$ - concrete's, steel's or carbon fibber's elasticity module; $U_{c}, U_{s}, U_{g}, U_{C F R P}$ - concrete's, steel's, glue's or carbon fiber's Poisson's ratio

In this article three comparative calculation methods are used: finite elements method, theory of multiple rods method and a method that is created by the authors which is based on iteration method.

\section{Finite elements method}

A three-dimensional calculation model from the provided test setup is made with the "ANSYS" program. Modelling the finite elements model the support point is chosen not at the end, but in the centre of the beam. This kind of scheme helps to avoid stress concentrators which distort the distribution of shear stress at the ends of the beam Fig. 3 . When creating the test setup and describing the bond between carbon fibre and concrete a layer of $1 \mathrm{~mm}$ thickness of glue was chosen. When changing the glue layer elasticity modulus, the bond between the layers changes as well as the values of bond stress. In this article the following glue elasticity modulus was used: $\mathrm{Eg}=0,2 \mathrm{GPa}$; 2,0 GPa; 28,0 GPa. The highest elasticity modulus (28 GPa), which equals to the concrete one, means that the bond between the layers is perfectly good. An elasticity modulus

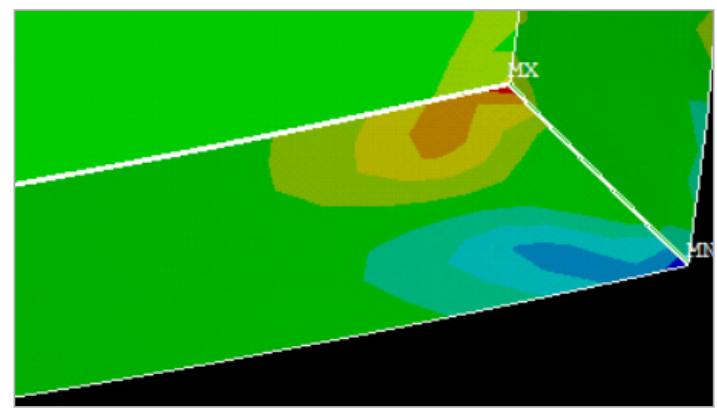

(2,0 GPa) represent the real glue elasticity modulus [Rabinovitch 2001, Adruini 1997]. Smallest elasticity modulus $(0,2 \mathrm{GPa})$ means that the bond between the layers is very poor. If the bond is even more reduced the stress in the concrete and the rebar does not change. In order to describe the concrete SOLID65 element was used. This element describes the nonlinear behaviour of a concrete. (ANSYS 2016) 


\section{Theory of multiple rods}

The theory of multiple rods was used to describe the behaviour of layered structures when there is a certain bond. The calculation method is used by (Ржаницын 1982, Ржаницын 1986). In Fig. 4 the certain geometrical parameters, bond stress and relative strains distribution in the contact zone are provided. The calculation formulas for the two layered structures are provided below.

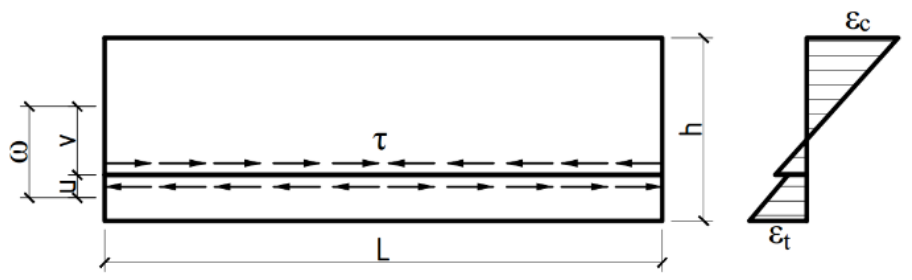

Fig. 4

Shear stress and relative strains distribution in the contact zone

The bond stress of the contact zone through the element length of is calculated by the following equation:

$$
\begin{aligned}
& \tau(x)=\frac{\Delta \cdot \lambda \cdot \sinh (\lambda \cdot \mathrm{x})}{\gamma \cdot \cosh \left(\lambda \cdot \frac{L}{2}\right)} \\
& \gamma=\frac{1}{E_{1} \cdot A_{1}}+\frac{1}{E_{2} \cdot A_{2}}+\frac{\omega^{2}}{E_{1} \cdot A_{1}+E_{2} \cdot A_{2}} \\
& \Delta=\frac{M_{E d} \cdot \omega}{E_{1} \cdot A_{1}+E_{2} \cdot A_{2}} \\
& \lambda=\sqrt{\xi} \cdot \gamma \\
& \xi=\xi_{\max } \cdot\left(2,5-1,5 \cdot \frac{E_{g}}{E_{c}}\right) \frac{E_{g}}{E_{c}}
\end{aligned}
$$

\section{Authors proposed calculation method}

The base of this proposed calculation method consists of iteration (layers) method (Zadlauskas 2013). From the values received using this method the distribution of bond stress through the length of the element is drawn. Iteration method is created when the cross-section of the element is divided into the finite numbers of layers Fig. 5. Every separate layer has its own stiffness and strains. All equations are written into the matrix form:

$$
[E] \cdot\{\varepsilon\}=\{F\}
$$

Relative strains, forces and elasticity module matrix:

$$
\varepsilon=\left\{\begin{array}{llllll}
\varepsilon_{C F R P}^{\prime} & \varepsilon_{1} & \varepsilon_{2} & \varepsilon_{3} & \ldots & \varepsilon_{i}
\end{array}\right\}
$$

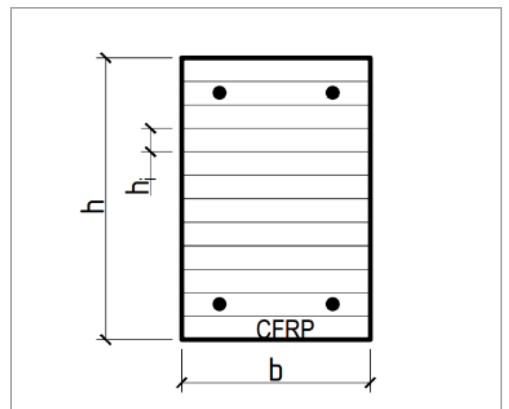

Fig. 5

Dividing of the cross-section in the layers 


\begin{tabular}{|c|c|c|c|c|c|c|c|c|}
\hline$F=\{0$ & 0 & $\begin{array}{llll}0 & 0 & \ldots\end{array}$ & 1\} & & & & & (8) \\
\hline$[E]=$ & $E_{C F R}$ & $\begin{array}{c}1 \\
0 \\
0 \\
\ldots \\
{ }_{P} A_{C F R P} k_{C F R P} \\
0\end{array}$ & $\begin{array}{c}-2 \\
1 \\
0 \\
\cdots \\
E_{c 1} A_{c 1} \\
E_{c 1} A_{c 1} h_{i}\end{array}$ & $\begin{array}{c}1 \\
-2 \\
1 \\
\ldots \\
E_{c 2} A_{c 2} \\
2 E_{c 2} A_{c 2} i\end{array}$ & $\begin{array}{c}0 \\
1 \\
-2 \\
\ldots \\
E_{c 3} A_{c 3} \\
3 E_{c 3} A_{c 3} h_{i}\end{array}$ & $\begin{array}{l}\cdots \\
\cdots \\
\cdots \\
\cdots \\
\cdots \\
\cdots\end{array}$ & $\left.\begin{array}{c}0 \\
0 \\
0 \\
0 \\
E_{c, i} A_{c, i} \\
n E_{c, i} A_{c, i} h_{i}\end{array}\right]$ & (6) \\
\hline
\end{tabular}

where: $k_{\text {CFRP }}$ - coefficient which evaluates the bond between carbon fibre and concrete, $A_{i}-$ cross-section area, $E_{i}$ - elasticity modulus of layer, $h_{i}$ - height of layer, $\varepsilon_{i}$ - relative strain, $M$ bending moment.

When the first iteration is done the relative strains of every layer are recalculated. If the relative strains exceed the elastic limit of this layer, then the elasticity modulus of this layer is reducing. If the relative strains exceed the ultimate strains, then the strain modulus of this layer is set to 0 . Sufficiently precise strains are received after calculating a couple of iterations. According to the received strains of layers and the strain modulus it is possible to easily calculate the missing parameters.

\section{Results}

\section{Fig. 6}

Shear stress through the length of the element when the bond between the elements is small $\left(E_{q}=0,2 \mathrm{GPa}\right)$

\section{Fig. 7}

Shear stress through the length of the element when the bond between the elements is medium $\left(E_{q}=2,0 \mathrm{GPa}\right)$
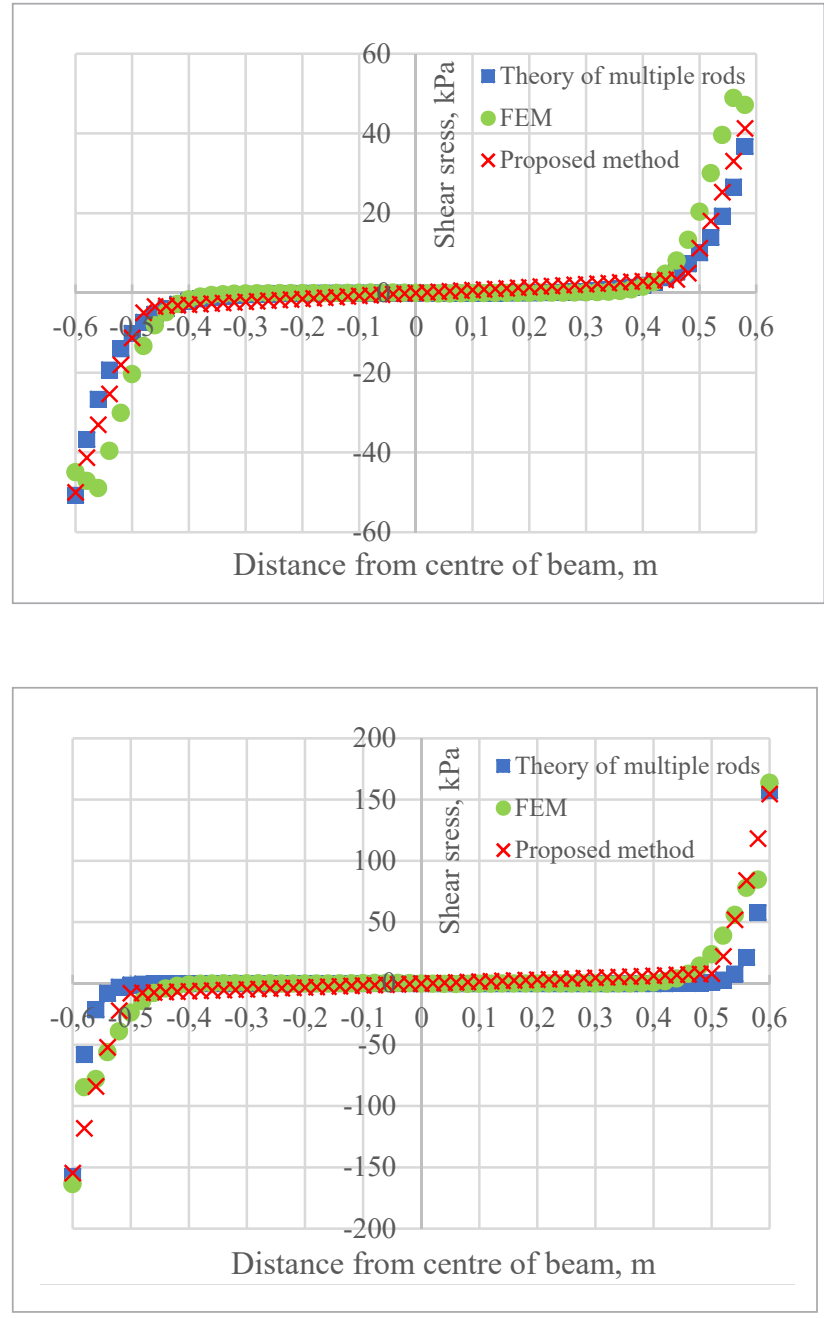

The results of the analysis are shown in Fig. 6 - Fig. 9. The maximum bonding coefficient according to the theory of multiple rods is $\xi_{\text {max }}$ $=4,69 \cdot 10^{11} \mathrm{~N} / \mathrm{m}^{2}$. This coefficient is received by the method of approximation and accepted as maximal. If this value would be increased the normal stress in the cross-section would remain as constant. According to the results in the diagrams it is seen that the maximum bond stress of the contact zone appears at the ends of the element and it distribute by parabola. Further from the end the bond stress decreases significantly and changes according to the line function.

Analysing the results according to FEM the uniqueness at the ends of the beam when the bond is small was noticed. Analysis of the shear stress obtained by FEM shows that the shear stress becomes decrease at the end of the beam. The maximum value of shear stress is reached not at the end of the structure, but a little bit before it. Such a distribution of stress is in- 
herent when the concrete reaches the marginal shear stress (Gou 2005, Gou 2007). According to the proposed method there is not obtained the decrease of stress.

In the Fig.9 the presented shear stress level is higher because of bigger bending moment that was assumed for the elastic plastic analysis. In Fig. 6-8 the normal stress in concrete are under elastic zone.

In Table 2 the maximum shear stress of a contact zone is provided. They were calculated by different methods evaluating various bonds. One case of calculation was chosen in order to evaluate the plastic behaviour of a concrete. In order to reach the plastic behaviour of a concrete it was needed to increase the bending moment of a beam. The tension strength of a concrete . It was accepted that the concrete strain modulus is varying linearly from the highest to zero (when a crack appears). This change is described in formulas $(7,8)$. Also, in this table the maximum deviation values of authors proposed method from FEM or the theory of multiple rods are provided. The maximum difference of authors proposed method did not exceed 5,9\%.

Maximum relative strains when the concrete is in elastic stage:

$$
\varepsilon_{c t, e l}=0,4 \cdot \frac{f_{c t}}{E_{c}}
$$

Maximum relative strains before the crack appears:

$$
\varepsilon_{c t, l i m}=2 \cdot \frac{f_{c t}}{E_{c}}
$$

According to the results obtained by finite elements method and the theory of multiple rods method, the formulas to calculate the

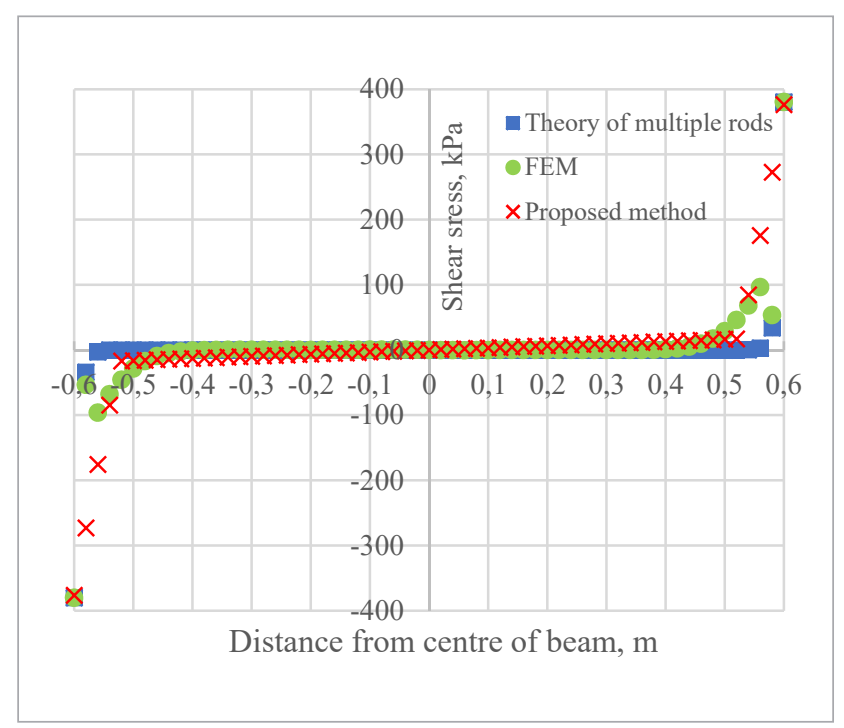

Fig. 8

Shear stress through the length of the element when the bond between the elements is perfect $\left(E_{g}=28 \mathrm{GPa}\right)$

\begin{tabular}{|c|c|c|c|c|}
\hline & $\tau_{F E M}, \mathrm{~Pa}$ & $\tau_{T M R}, P a$ & $\tau_{P M}, P a$ & $\begin{array}{c}\text { Max } \\
\text { difference, \% }\end{array}$ \\
\hline Poor bond & $4,89 \cdot 10^{4}$ & $5,07 \cdot 10^{4}$ & $5,00 \cdot 10^{4}$ & 2,16 \\
\hline Medium bond & $1,64 \cdot 10^{5}$ & $1,57 \cdot 10^{5}$ & $1,55 \cdot 10^{5}$ & 5,90 \\
\hline Perfect bond & $3,80 \cdot 10^{5}$ & $3,80 \cdot 10^{5}$ & $3,76 \cdot 10^{5}$ & 1,01 \\
\hline $\begin{array}{l}\text { Medium bond } \\
\text { (Plastic } \\
\text { behaviour of } \\
\text { concrete is } \\
\text { evaluated) }\end{array}$ & $5,73 \cdot 10^{5}$ & - & $5,69 \cdot 10^{5}$ & 0,71 \\
\hline
\end{tabular}

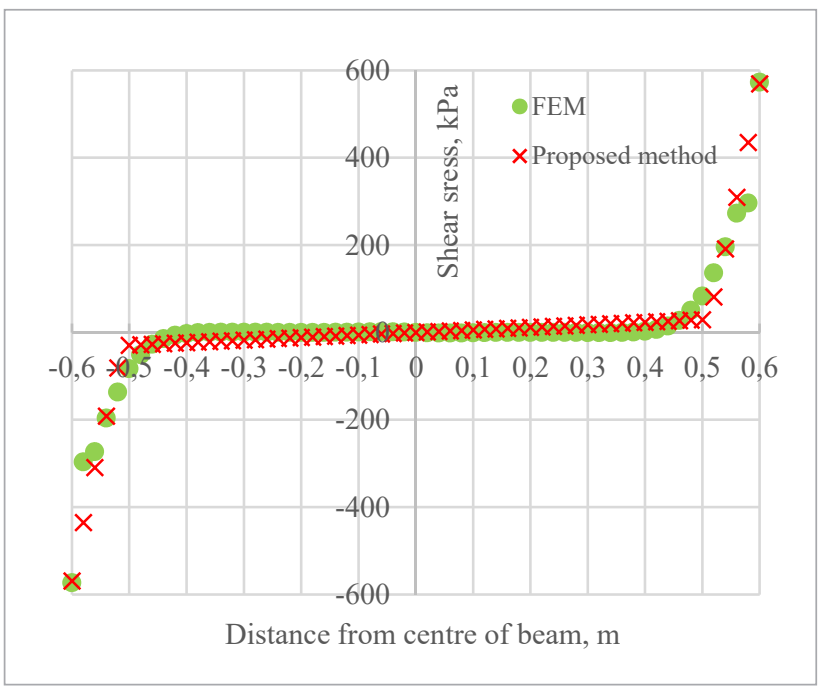

\section{Fig. 9}

Shear stress through the length of the element when the bond between the stress is medium $\left(E_{g}=2,0 \mathrm{GPa}\right.$ ) (plastic behaviour of concrete is evaluated)

\section{Table 2}

Maximum shear stress 
shear stress through the element length were derived. Coefficient evaluating the bond between the concrete and the carbon fibre:

$$
k_{C F R P}=\sqrt[3]{\frac{\xi}{\xi_{\max }}}
$$

Analysing the bond between the concrete and the carbon fibre it was noticed that the maximum shear stress are received at the ends of structures and directly depends on normal stress of the fibre:]

$$
\tau_{\max }=\frac{k_{C F R P}+0,33}{10} \cdot k_{C F R P} \cdot \varepsilon_{C F R P} \cdot E_{C F R P}
$$

Shear stress at the ends of an element are distributed by a principle of parabola and could be calculated by the following formulas:

$$
\begin{aligned}
& \tau=a \cdot x^{2}+b \cdot x \\
& a=\frac{\tau_{\max }}{\left(\frac{L}{2}\right)^{2}-\frac{L}{2} \cdot x_{2}} \\
& b=-a \cdot x_{2} \\
& x_{2}=\frac{L}{2} \cdot 0,9-\frac{0,02}{k_{C F R P}}
\end{aligned}
$$

Distance from the end of element where the parabola equation of shear stress is valid:

$$
l_{0}=0,9 \cdot\left(\frac{L}{2}-x_{2}\right), \quad \text { but } l_{0} \geq 0
$$

Discussion

\section{Conclusions}

\section{Acknow- ledgment}

The analysis was made for simple supported beam Fig. 1 loaded by bending moment at the ends. For different loading cases (if the form of moments diagram change) the additional analysis has to be made and the proposed formulas have to be checked additionally. The plastic behaviour of concrete was evaluated by the proposed method and the FEM but not by the theory of multiple rods

1 The difference of values of shear stress using the proposed method did not exceed 5,9\% compared to FEM and the theory of multiple rods.

2 Maximum shear stress of contact zone obtained at

$\angle$ the ends of a beam or close to it.

3 The shear stress in contact zone at the ends of a beam, according to the proposed method, 3 distributes by a parabola function.

Authors proposed method is possible to be used for calculation of the reinforced concrete 4 structures strengthened by carbon fibre at the elastic plastic stress stage of concrete.

The authors thank to Justinas Valeika for helping on English. 
Guo Z.G., Cao S.Y., Sun W.M., Lin X.Y. Experimental study on bond stress-slip behaviour between FRP sheets and concrete. Proceedings of international symposium on bond behaviour of FRP in structures. 2005;(Bbfs):77-84.

GuoD. J. Interfacial models for fiber reinforced polymer (FRP) sheets externally bonded to concrete. Michigan state university, 2007.

Lorenzis D., Miller B., Nanni A. Bond of FRP laminates to concrete.ACl Materials Journal, 2001;98(3):256-264.

Zabulionis D. Stress and strain analysis of a bilayer composite beam with interlayer slip under hygrothermal loads. Mechanika, 2005;6(6):5-12.

The program “ANSYS”. Mechanical APDL17.0. 2016. Rabinovitch O., Frostig Y. Nonlinear high-order anal- ysis of cracked rc beams strengthened with FRP strips. Journal of Structural Engineering. 2001; 127 (April): 381-389.

Adruini M., Nanni A. Behavior of precracked RC beams strengthened with carbon FRP sheets. Journal of composites for construction.1997; May: 63-70.

Ржаницын, А. Р. Составныестержни и пластинки [Composite of rods and plates]. Стройиздат. 1986, 316.

Ржаницын, А. Р. Строительнаямеханика [Structural mechanics]. Высшаяшкола. 1982, 396.

Zadlauskas S. Gelžbetoninių tiltų pleišejimo ir deformacijų nuo statinių ir dinaminių apkrovų tyrimai [The reserarch on cracking and deformation of reinforced concrete bridges under static and dynamic loads]. Doctoral dissertation, Kauans; 2013; 113 p.

\section{References}

\section{TADAS LISAUSKAS}

\section{PhD student}

Kaunas University of Technology, Faculty of Civil Engineering and Architecture, Department of Civil Engineering and Architecture competences center

\section{Main research area}

FRP Strengthened Reinforced Concrete Structures

\section{Address}

Studentu st. 48,

LT-51367 Kaunas, Lithuania

Tel. 8-610-29-841

E-mail: tadaslisauskas@gmail. com

\section{MINDAUGAS AUGONIS Assoc. Prof \\ Kaunas University of Technology, Faculty of Civil Engineering and Architecture, Department of Civil Engineering and Architecture competences center \\ Main research area \\ Durability of Engineering Structures, Strength and Stability or Reinforced Concrete}

\section{Address}

Studentu st. 48 LT-51367 Kaunas, Lithuania

\section{ŠARŪNAS KELPŠA About the Lecturer Authors}

Kaunas University of Technology, Faculty of Civil Engineering and Architecture, Department of Civil Engineering and Architecture competences center

\section{Main research area}

Behaviour and analysis of reinforced concrete, steel and composite structures

\section{Address}

Studentu st. 48, LT-51367 Kaunas, Lithuania 